\title{
Concomitant existence and interaction of cardiovascular abnormalities in obstructive sleep apnea subjects with normal clinic blood pressure
}

\author{
Hirofumi Tomiyama, Yoshifumi Takata, Kazuki Shiina, Chisa Matsumoto, Jiko Yamada, Masanobu Yoshida \\ and Akira Yamashina
}

This study was conducted to examine the existence of increased arterial stiffness of the central to middle-sized arteries and left ventricular (LV) structural/functional abnormalities in subjects with obstructive sleep apnea (OSA) who had normal clinic blood pressure. Brachial-ankle pulse wave velocity (baPWV) measurement and echocardiography were conducted in 164 consecutive subjects with normal clinic blood pressure evaluated for sleep disorders. Multivariate linear regression analysis showed that the apnea-hypopnea index (AHI) was an independently associated variable, even after adjustments for age, gender and risk factors for cardiovascular disease, with baPWV $\left(R^{2}=0.39, \beta=0.19, P<0.01\right)$ and the E/A ratio $\left(R^{2}=0.51, \beta=-0.27, P<0.01\right)$. The baPWV and LV relative wall thickness at diastole (RWTd) were significantly higher $(P<0.05)$, and the $E / A$ ratio was significantly lower $(P<0.05)$, in subjects with severe OSA (AHI $\geqslant 30$ episodes per hour) than in non-OSA subjects $(A H I<5$ episodes per hour). The analysis also showed that the baPWV bore a significant independent relationship to the RWTd $(\beta=0.19, P=0.02)$ and E/A ratio $(\beta=-0.12, P=0.04)$ adjusted for the risk factors for cardiovascular disease, the presence of metabolic syndrome and the severity of OSA. Thus, in this study, we showed the existence of increased arterial stiffness of the central to middle-sized arteries and LV structural/functional abnormalities in severe OSA patients, even in subjects with normal clinic blood pressure, suggesting the possible existence of a direct association between OSA and cardiovascular abnormalities.

Hypertension Research (2009) 32, 201-206; doi:10.1038/hr.2008.32; published online 23 January 2009

Keywords: arterial stiffness; left ventricular remodeling; sleep apnea

\section{INTRODUCTION}

An increasing body of evidence has been collected to show that obstructive sleep apnea (OSA) is a risk factor for cardiovascular diseases. ${ }^{1,2}$ Some studies have reported a high prevalence of increased arterial stiffness, ${ }^{3,4}$ and others have reported a high prevalence of left ventricular (LV) structural and functional abnormalities (that is, increased LV mass, LV concentric remodeling and LV diastolic dysfunction) in subjects with OSA., ${ }^{5}$ These abnormalities are markers of future cardiovascular events and/or prognosis, and are thought to contribute to cardiovascular risk through several mechanisms. ${ }^{7,8}$ However, a few studies have reported the concomitant existence of both abnormalities (that is, vascular abnormalities and cardiac abnormalities). ${ }^{9}$ Hypertension is frequently associated with OSA, ${ }^{10,11}$ and may be a common causal factor for the cardiovascular abnormalities in these patients. ${ }^{4,5,10,11}$

On the other hand, recent studies have showed an increased LV mass and an impaired LV diastolic function even in normotensive subjects with OSA. ${ }^{5,12}$ Obesity is frequently associated with OSA $^{1,2,10,11}$ and, besides elevation of blood pressure, other obesityrelated atherogenic factors (that is, obesity itself, dyslipidemia and abnormal glucose metabolism), intermittent hypoxia producing sympathetic activation and inflammation and/or increased oxidative stress may also contribute to increased arterial stiffness and LV structural/ functional abnormalities in subjects with OSA. ${ }^{1,2,10,11}$ In addition, an increase in aortic impedance caused by increased arterial stiffness may give rise to structural/functional abnormalities of the LV, referred to as arterial-ventricular coupling. ${ }^{13,14}$ However, it has not yet been clarified whether OSA might be associated with increased arterial stiffness and cardiac structural/functional abnormalities, even in subjects with normal clinic blood pressure.

This study was conducted to examine the existence of increased arterial stiffness of the central to middle-sized arteries and LV structural/functional abnormalities in OSA subjects with normal clinic blood pressure.

\section{METHODS}

Patients

We evaluated all eligible patients from 579 consecutive patients ( 488 men, 91 women; $53 \pm 15$ years), who underwent overnight polysomnography at the 
Tokyo Medical University Hospital from November 2005 to September 2007. The exclusion criteria were as follows: the presence of cardiovascular diseases (ischemic heart disease, valvular heart disease, cardiomyopathy, atrial fibrillation, stroke, arteriosclerosis obliterance, aortic aneurythm, pulmonary embolism and an LV ejection fraction of less than 50\%) $(n=115)$, renal insufficiency (serum creatinine level $\left.>91.5 \mu \mathrm{moll}^{-1}\right) \quad(n=8)$, medication for dyslipidemia and/or diabetes mellitus $(n=114)$ and patients taking steroids $(n=3)$. The blood pressure classification was conducted based on the average blood pressure readings obtained from two clinic visits. The mean blood pressure was calculated as follows: the average diastolic blood pressure+(the average systolic blood pressure-the average diastolic blood pressure)/3. Subjects who were on antihypertensive medication and/or whose blood pressure was $\geqslant 140 / 90 \mathrm{~mm} \mathrm{Hg}$ were defined as subjects with hypertension, and these subjects were excluded $(n=175)$. Finally, 164 subjects were selected for this study (149 men, 15 women; age, $48 \pm 11$ years), which was conducted with the approval of the Ethics Committee of Tokyo Medical University, and written informed consent was obtained from each of the subjects before their participation.

\section{Sleep study}

Overnight, fully attended polysomnography monitoring was performed with the Alice 4 Sleep System (Respironics Inc., Murrysville, PA, USA) in the sleep laboratory. The sleep stages were monitored by electroencephalography, electrooculography and electromyography, according to standard criteria, ${ }^{15,16,17}$ and arousals were defined according to the standard criteria of the American Academy of Sleep Medicine. ${ }^{15,16,17}$ Oronasal airflow was measured with a thermistor, thoracoabdominal movements were monitored with a strain gauge and the oxyhemoglobin saturation in the blood was monitored by pulse oximetry. The respiratory events were scored manually. Apnea was defined as cessation of oronasal airflow for $10 \mathrm{~s}$ or more, and hypopnea was defined as a $50 \%$ or more decrease in oronasal airflow lasting for more than $10 \mathrm{~s}$, associated with a $3 \%$ or more decrease in arterial oxygen saturation relative to the baseline level. Obstructive apnea/hypopnea was defined as the significant apnea/hypopnea in the presence of thoracoabdominal effort, and central apnea/hypopnea was defined as significant apnea/hypopnea in the absence of oronasal air flow or thoracoabdominal effort. ${ }^{15,16,17}$ The apnea-hypopnea index (AHI) was calculated as the total number of apnea and hypopnea episodes per hour of sleep. The patient was defined as having OSA when the obstructive component was dominant and the AHI was $\geqslant 5$ per hour. The severity of OSA was classified according to the criteria of the American Academy of Sleep Medicine. ${ }^{16}$ Epworth sleepiness scale was used to evaluate daytime sleepiness. ${ }^{18}$

\section{Echocardiogram}

Before polysomnography, all the patients underwent transthoracic echocardiography (Sequoia Siemens Medical Solutions USA Inc., Mountain View, CA, USA). The LV internal dimensions and wall thicknesses were measured at end diastole and end systole. Both two-dimensional and M-mode echocardiography were performed in all the patients. The end-diastolic and end-systolic dimensions were obtained from the M-mode recordings. Thereafter, end-diastolic LV volume and end-systolic LV volume were derived by Teichholtz's methods, ${ }^{19}$ and LV ejection fraction was calculated. The LV mass was calculated according to the anatomically validated formula of Devereux. ${ }^{20}$ The LV mass index was calculated by dividing the LV mass by the body surface area. The interobserver and intraobserver coefficients of variation for the measurement of the LV mass were 7.2 and $13.5 \%$, respectively. The relative wall thickness was calculated as $2 \times \mathrm{LV}$ posterior wall thickness at diastole/LV diameter at diastole. ${ }^{13,21}$ Pulsed Doppler measurements of the LV diastolic inflow were obtained under twodimensional echo guidance. In brief, the LV diastolic filling pattern was recorded with the transducer in the apical position of the heart, with the patient in the partial left lateral decubitus position, during apnea on expiration and sampling between the mitral leaflet tips. The peak velocity of early rapid filling (E velocity) and that of atrial filling (A velocity) were recorded, and the $\mathrm{E} / \mathrm{A}$ ratio and the deceleration time, calculated as the interval from the E-wave peak to the decline of the velocity to baseline values, were obtained from three consecutive cardiac cycles. All the subjects in this study had a normal systolic cardiac function and none of the subjects had pseudonormal cardiac diastolic function (E/A ratio of 1.0-1.5 and/or deceleration time of $>240 \mathrm{~ms}$ ). ${ }^{22}$ The interobserver and intraobserver coefficients of variation for the Doppler velocity measurements were 4.8 and $4.1 \%$, respectively.

\section{Brachial-ankle pulse wave velocity}

The brachial-ankle pulse wave velocity (baPWV) was measured using a volume-plethysmographic apparatus (Form/ABI; Colin Co. Ltd, Komaki, Japan), in accordance with a methodology described earlier. ${ }^{23}$ Briefly, electrocardiographic electrodes were placed on both wrists, and a microphone for the phonocardiogram was attached to the left chest. ECGs and phonocardiograms were used to provide timing markers for the device. Occlusion cuffs, which were connected to both the plethysmographic and oscillometric sensors, were tied around both the upper arms and ankles while the subjects lay in the supine position. The brachial and post-tibial arterial pressures were measured by the oscillometric sensor. The brachial and post-tibial arterial pressure waveforms, determined by the plethysmographic sensor and recorded for $10 \mathrm{~s}$, were stored.

Table 1 Clinical characteristics of study subjects

\begin{tabular}{lc}
\hline Age (years) & $48 \pm 11$ \\
Gender (male/female) & $149 / 15$ \\
Smoke $(\%)$ & $88(54)$ \\
BMI $\left(\mathrm{kg} \mathrm{m}^{-2}\right)$ & $25.9 \pm 3.6$ \\
Waist $(\mathrm{cm})$ & $90 \pm 10$ \\
SBP $(\mathrm{mm} \mathrm{Hg})$ & $119 \pm 10$ \\
DBP $\left(\mathrm{mm} \mathrm{Hg}^{2}\right)$ & $72 \pm 7$ \\
MBP (mm Hg) & $88 \pm 8$ \\
LDL (mmol I-1) & $3.3 \pm 0.8$ \\
HDL (mmol $\left.{ }^{-1}\right)$ & $1.3 \pm 0.3$ \\
TG (mmol I-1) & $2.0 \pm 1.3$ \\
FPG (mmol I-1) & $5.0 \pm 0.8$ \\
Crnn ( $\left.\mu \mathrm{mol} \mathrm{I}^{-1}\right)$ & $71 \pm 12$ \\
MetS (\%) & $27(17)$ \\
AHI (per hour) & $35 \pm 22$ \\
ODI (per hour) & $29 \pm 23$ \\
ARI (per hour) & $39 \pm 19$ \\
ESS score & $10 \pm 5$ \\
LowSpO $(\%)$ & $80 \pm 9$
\end{tabular}

\begin{tabular}{|c|c|}
\hline \multicolumn{2}{|l|}{ Sleep stage (\%) } \\
\hline REM sleep & $18.8 \pm 5.6$ \\
\hline Stage I sleep & $26.5 \pm 13.5$ \\
\hline Stage II sleep & $50.5 \pm 12.4$ \\
\hline Stage III sleep & $3.7 \pm 4.3$ \\
\hline Stage IV sleep & $0.5 \pm 1.3$ \\
\hline baPWV $\left(\mathrm{cm} \mathrm{s}^{-1}\right)$ & $1385 \pm 223$ \\
\hline $\mathrm{LAD}(\mathrm{mm})$ & $41.9 \pm 2.4$ \\
\hline LVDd (mm) & $48.1 \pm 3.6$ \\
\hline LVDs (mm) & $30.5 \pm 2.9$ \\
\hline LVSTd (mm) & $9.7 \pm 1.2$ \\
\hline PWTd (mm) & $9.8 \pm 1.2$ \\
\hline LVMI $\left(\mathrm{g} \mathrm{m}^{-2}\right)$ & $113 \pm 22$ \\
\hline RWTd & $0.41 \pm 0.5$ \\
\hline LVEF (\%) & $66 \pm 4$ \\
\hline E/A ratio & $1.2 \pm 0.4$ \\
\hline DCT (ms) & $200 \pm 35$ \\
\hline
\end{tabular}

Abbreviations: AHI, apnea-hypopnea index; ARI, arousal index; baPWV, brachial-ankle pulse wave velocity; BMI, body mass index; Crnn, serum creatinine; DBP, diastolic blood pressure DCT, deceleration time; ESS, Epworth sleepiness scale; FPG, fasting plasma glucose; HDL, serum high-density lipoprotein cholesterol; LVSTd, left ventricular septal thickness at diastole; $\mathrm{LAD}$, left atrial diameter; LDL, serum low-density lipoprotein cholesterol; $\mathrm{LowSpO}_{2}$, the lowest oxygen saturation during sleep; LVDd, left ventricular diameter at diastole; LVDs, left ventricular diameter at systole; LVEF, left ventricular ejection fraction; LVMI, left ventricular mass index; MetS, number of subjects with metabolic syndrome; ODI, oxygen desaturation index; PWTd, left MetS, number of subjects with metabolic syndrome; ODI, oxygen desaturation index; PWTd, left
ventricular posterior wall thickness at diastole; RWTd, relative wall thickness at diastole; SBP, systolic blood pressure; smoke, number of smokers; TG, serum triglycerides; waist, waist circumference. 
Table 2 The correlation coefficient in univariate linear regression analysis to assess the relationship of severity of obstructive sleep apnea (apnea-hypopnea index) with the variables of cardiovascular abnormalities

\begin{tabular}{lccccc}
\hline Dependent variable & AHI & ODI & ARI & Waist & BMI \\
\hline baPWV & $0.29^{*}$ & $0.27^{*}$ & $0.25^{*}$ & $0.25^{*}$ & 0.15 \\
LVMI & 0.08 & 0.05 & 0.08 & $0.16^{*}$ & 0.15 \\
RWTd & $0.25^{*}$ & $0.25^{*}$ & $0.18^{*}$ & $0.31^{*}$ & $0.31^{*}$ \\
LVEF & 0.10 & 0.09 & 0.07 & 0.09 & 0.10 \\
E/A ratio & $-0.41^{*}$ & $-0.38^{*}$ & $-0.33^{*}$ & $-0.36^{*}$ & $-0.26^{*}$ \\
DCT & 0.03 & 0.01 & 0.05 & 0.06 & 0.01 \\
SBP & $0.16^{*}$ & 0.14 & 0.13 & $0.23^{*}$ & $0.23^{*}$ \\
DBP & 0.14 & 0.14 & 0.11 & $0.20^{*}$ & 0.08 \\
MBP & $0.16^{*}$ & $0.16^{*}$ & 0.13 & $0.23^{*}$ & $0.16^{*}$ \\
\hline
\end{tabular}

Abbreviations: AHI, apnea-hypopnea index; ARI, arousal index; baPWV, brachial-ankle pulse wave velocity; BMI, body mass index; DBP, diastolic blood pressure; DCT, deceleration time; LVEF, left ventricular ejection fraction; LVMI, left ventricular mass index; MBP, mean blood pressure; ODI, oxygen desaturation index; RWTd, relative wall thickness at diastole; SBP, systolic blood pressure; waist, waist circumference.

${ }^{*} P<0.01$.

Table 3 The results of multivariate linear regression analysis to assess the significance of the relationship of the severity of obstructive sleep apnea (apnea-hypopnea index) and that of mean blood pressure with arterial stiffness and left ventricular structural and functional abnormalities with adjustment (adjusted for age, gender, smoking, body mass index, serum low-density lipoprotein cholesterol, serum high-density lipoprotein cholesterol, serum triglycerides, fasting plasma glucose and the presence of metabolic syndrome)

\begin{tabular}{lllrrr}
\hline Dependent variable & $\mathrm{R}^{2}$ & Parameter & $\beta$ & t-value & P-value \\
\hline baPWV & \multirow{2}{*}{0.39} & $\mathrm{AHI}$ & 0.19 & 2.50 & $<0.01$ \\
& & $\mathrm{MBP}$ & 0.31 & 3.90 & $<0.01$ \\
LVMI & 0.31 & $\mathrm{AHI}$ & -0.08 & -0.94 & 0.35 \\
& & $\mathrm{MBP}$ & 0.06 & 0.72 & 0.47 \\
RWTd & 0.32 & $\mathrm{AHI}$ & 0.05 & 0.60 & 0.55 \\
& & $\mathrm{MBP}$ & 0.05 & 0.64 & 0.53 \\
LVEF & 0.17 & $\mathrm{AHI}$ & 0.16 & 1.96 & 0.09 \\
& & $\mathrm{MBP}$ & 0.15 & 1.60 & 0.11 \\
E/A ratio & 0.51 & $\mathrm{AHI}$ & -0.29 & -4.16 & $<0.01$ \\
& & $\mathrm{MBP}$ & -0.03 & -0.45 & 0.66 \\
DCT & \multirow{2}{*}{0.20} & AHI & -0.01 & -0.01 & 0.98 \\
& & MBP & -0.06 & -0.65 & 0.52 \\
\hline
\end{tabular}

Abbreviations: AHI, apnea-hypopnea index; baPWV, brachial-ankle pulse wave velocity; DCT, deceleration time; LVEF, left ventricular ejection fraction; LVMI, left ventricular mass index; MBP, mean blood pressure; RWTd, relative wall thickness at diastole.

The measurements were taken after the subjects had rested for at least $5 \mathrm{~min}$ in the supine position, in an air-conditioned room $\left(24-26^{\circ} \mathrm{C}\right)$ earmarked exclusively for this purpose. In 55 volunteers, the intraclass correlation coefficient of reproducibility of the baPWV was $0.92 .^{23}$ The blood pressure determined by the oscillometric sensor and the heart rate were simultaneously obtained during measurement of the baPWV.

\section{Laboratory measurements}

The serum levels of low-density lipoprotein cholesterol, high-density lipoprotein cholesterol, triglycerides and creatinine, and the plasma level of glucose were measured enzymatically in the subjects after they had fasted overnight.

We adopted the criteria of the Japanese Expert Committee on the Diagnosis and Classification of Metabolic Syndrome ${ }^{24}$ for the clinical recognition of
Table 4 Clinical characteristics of the three subject groups classified by the severity of sleep apnea in subjects without hypertension

\begin{tabular}{lccc}
\hline & Non $(\mathrm{n}=14)$ & MiMo $(\mathrm{n}=65)$ & $\operatorname{Sev}(\mathrm{n}=85)$ \\
\hline Age (years) & $44 \pm 10$ & $46 \pm 11$ & $49 \pm 11$ \\
Gender (male/female) & $12 / 2$ & $59 / 6$ & $78 / 7$ \\
Smoke (\%) & $5(36)$ & $33(51)$ & $50(58)$ \\
BMI (kg m$)^{-2}$ ) & $23.6 \pm 2.8$ & $25.0 \pm 3.1$ & $27.0 \pm 3.7^{* \dagger}$ \\
Waist (cm) & $82 \pm 9$ & $86 \pm 8^{*}$ & $94 \pm 9^{* \dagger}$ \\
SBP (mm Hg) & $119 \pm 14$ & $118 \pm 9$ & $121 \pm 10^{\dagger}$ \\
DBP (mm Hg) & $72 \pm 9$ & $71 \pm 7$ & $73 \pm 7$ \\
MBP (mm Hg) & $87 \pm 10$ & $87 \pm 7$ & $89 \pm 8$ \\
LDL (mmol I-1) & $3.0 \pm 0.4$ & $3.2 \pm 0.7$ & $3.4 \pm 0.9^{*}$ \\
HDL (mmol I-1) & $1.5 \pm 0.3$ & $1.3 \pm 0.4$ & $1.3 \pm 0.3$ \\
TG (mmol I-1) & $1.7 \pm 1.0$ & $1.9 \pm 1.1$ & $2.1 \pm 1.4$ \\
FPG (mmol I-1) & $4.7 \pm 0.4$ & $5.0 \pm 0.9$ & $5.1 \pm 0.9$ \\
Crnn ( $\mu \mathrm{mol} \mathrm{I}^{-1}$ ) & $67 \pm 9$ & $73 \pm 11^{*}$ & $71 \pm 12^{*}$ \\
MetS (\%) & $2(14)$ & $6(9)$ & $19(22)^{\dagger}$ \\
AHI (per hour) & $2.1 \pm 1.2$ & $19.7 \pm 6.1^{*}$ & $51.2 \pm 18.1^{* \dagger}$ \\
ODI (per hour) & $3.1 \pm 2.2$ & $12.6 \pm 4.9^{*}$ & $44.6 \pm 20.0^{* \dagger}$ \\
ARI (per hour) & $17 \pm 6$ & $28 \pm 10^{*}$ & $51 \pm 18^{* \dagger}$ \\
ESS score & $8 \pm 5$ & $10 \pm 4$ & $11 \pm 5$ \\
LowSpO $(\%)$ & $91 \pm 5$ & $85 \pm 6^{*}$ & $76 \pm 9 * \dagger$ \\
& & &
\end{tabular}

\begin{tabular}{lccc} 
Sleep stage (\%) & & & \\
REM sleep & $20.8 \pm 3.6$ & $19.6 \pm 5.5$ & $17.8 \pm 5.8$ \\
Stage I sleep & $14.9 \pm 5.6$ & $20.0 \pm 8.4$ & $33.2 \pm 13.9^{* \dagger}$ \\
Stage II sleep & $59.6 \pm 7.9$ & $54.9 \pm 10.3$ & $45.8 \pm 12.5^{* \dagger}$ \\
Stage III sleep & $3.9 \pm 4.8$ & $4.7 \pm 4.6$ & $2.9 \pm 3.9^{\dagger}$ \\
Stage IV sleep & $0.8 \pm 1.6$ & $0.8 \pm 1.6$ & $0.3 \pm 1.0^{\dagger}$ \\
LAD (mm) & $41.7 \pm 3.9$ & $41.9 \pm 2.9$ & $42.0 \pm 2.8$ \\
LVDd (mm) & $48.6 \pm 3.2$ & $48.2 \pm 3.4$ & $48.0 \pm 3.7$ \\
LVDs (mm) & $30.9 \pm 2.1$ & $30.7 \pm 2.6$ & $30.2 \pm 3.2$ \\
IVSTd (mm) & $9.3 \pm 1.2$ & $9.5 \pm 1.1$ & $9.9 \pm 1.2$ \\
PWTd (mm) & $8.9 \pm 0.8$ & $9.7 \pm 1.1^{*}$ & $10.0 \pm 1.2^{* \dagger}$ \\
\hline
\end{tabular}

Abbreviations: AHI, apnea-hypopnea index; ARI, arousal index; baPWV, brachial-ankle pulse wave velocity; BMI, body mass index; Crnn, serum creatinine; DBP, diastolic blood pressure wave velocity; BMI, body mass index; Crnn, serum creatinine; DBP, diastolic blood press
ESS, Epworth sleepiness scale; FPG, fasting plasma glucose; HDL, serum high-density lipoprotein cholesterol; LVSTd, left ventricular septal thickness at diastole; LAD, left atrial diameter; LDL, serum low-density lipoprotein cholesterol; $\mathrm{LowSpO}_{2}$, the lowest oxygen saturation during sleep; LVDd, left ventricular diameter at diastole; LVDs, left ventricular diameter at systole; LVEF, left ventricular ejection fraction; MiMo, subjects with mild-tomoderate obstructive sleep apnea; Non, subjects without obstructive sleep apnea; ODI, oxygen desaturation index; PWTd, left ventricular posterior wall thickness at diastole; SBP, systolic blood pressure; Sev, subjects with severe obstructive sleep apnea; smoke, number of smokers; blood pressure; Sev, subjects with severe obstructive
TG, serum triglycerides; waist, waist circumference.

TG, serum triglycerides; waist, waist circumference.
${ }^{*} P<0.05$ vs subjects without obstructive sleep apnea (assessed by analysis of variance); ${ }^{\dagger} P<0.05$ vs subjects with mild-to-moderate obstructive sleep apnea (assessed by analysis of variance).

metabolic syndrome in this study, namely, waist circumference $\geqslant 85 \mathrm{~cm}$, plus at least two of three risk factors: dyslipidemia (triglycerides $\geqslant 1.70 \mathrm{mmoll}^{-1}$ and/ or high-density lipoprotein cholesterol $<1.03 \mathrm{mmoll}^{-1}$ ), raised blood pressure (blood pressure $\geqslant 130 / 85 \mathrm{~mm} \mathrm{Hg}$ ) and raised plasma glucose (fasting plasma glucose $\left.\geqslant 6.11 \mathrm{mmoll}^{-1}\right)$.

Statistical analysis

Results were expressed as means \pm s.d. Figures are shown with error bars. The differences in each variable among the groups were evaluated by the un-paired $t$-test or by one-way analysis of variance for continuous variables and the $\chi^{2}$ test for categorical variables. In addition, the differences among the normotensive subjects, classified by the severity of OSA, were also assessed by analysis of covariance after adjustments. Univariate and multivariate linear regression analyses were applied for the assessment of the relationships among variables. The computations were performed using the SPSS-PC+ computer program (version 11.0; SPSS, Chicago, IL, USA). 


\section{RESULTS}

Table 1 shows the clinical characteristics of the subjects with normal office blood pressure. Table 2 shows the results of univariate linear regression analysis to assess the relationship of the severity of OSA with cardiovascular abnormalities. Univariate linear regression analysis showed that the markers of the severity of OSA correlated significantly with the baPWV, relative wall thickness at diastole (RWTd) and the E/A ratio. Of those markers, AHI had better correlations with the abnormalities than the other two (Table 2). Table 3 depicts the results of multivariate linear regression analysis to assess the significance of the relationship of AHI and that of mean blood pressure with arterial stiffness and LV structural/functional abnormalities, independent of age, gender, risk factors for cardiovascular disease (that is, smoking status, body mass index, serum low-density lipoprotein cholesterol, serum high-density lipoprotein cholesterol, serum triglycerides and fasting plasma glucose) and the presence of metabolic syndrome. AHI showed a significant relationship with the baPWV and the E/A ratio even after adjustments.

The subjects were divided into three groups, according to the severity of OSA, as follows: non-OSA: AHI $<5$ episodes per hour $(n=14)$; mild-to-moderate OSA: five episodes per hour $\leqslant \mathrm{AHI}<30$ episodes per hour $(n=65)$; and severe OSA: AHI $\geqslant 30$ episodes per hour $(n=85)$. Table 4 shows the clinical characteristics of these three groups of normotensive subjects. Age, body mass index, waist circumference and blood pressure were higher, and the serum highdensity lipoprotein cholesterol was lower, in subjects with severe OSA as compared with those without OSA (non-OSA) (Table 4). The baPWV and the RWTd were higher, and the E/A ratio was lower, in subjects with severe OSA than in non-OSA subjects, without adjustment. These findings, except the RWTd, were significant even after adjustments for age, gender, risk factors for cardiovascular disease and the presence of metabolic syndrome (Figure 1).

As shown in Table 5, multivariate linear regression analysis showed that the baPWV bore a significant relationship with the RWTd and the E/A ratio independent of the severity of OSA (AHI), other adjusted variables (age, gender and risk factors for cardiovascular disease) and the presence of metabolic syndrome.

Table 5 The results of multivariate linear regression analysis to assess the significance of the relationship of brachial-ankle pulse wave velocity with the parameters of LV structural and functional abnormalities adjusted for the severity of obstructive sleep apnea (apnea-hypopnea index) and other variables (age, gender, smoking, body mass index, mean blood pressure, serum low-density lipoprotein cholesterol, serum high-density lipoprotein cholesterol, serum triglycerides, fasting plasma glucose and the presence of metabolic syndrome)

\begin{tabular}{lcrrr}
\hline Explained variable & $\mathrm{R}^{2}$ & $\beta$ & $\mathrm{t}$-value & P-value \\
\hline LVMI & 0.31 & -0.01 & -0.01 & 0.99 \\
RWTd & 0.35 & 0.19 & 2.30 & 0.02 \\
LVEF & 0.18 & -0.64 & -0.98 & 0.25 \\
E/A ratio & 0.52 & -0.12 & -1.99 & 0.04 \\
DCT & 0.20 & -0.02 & -0.21 & 0.83
\end{tabular}

Abbreviations: DCT, deceleration time; LVEF, left ventricular ejection fraction; LVMI, left ventricular mass index; RWTd, relative wall thickness at diastole.
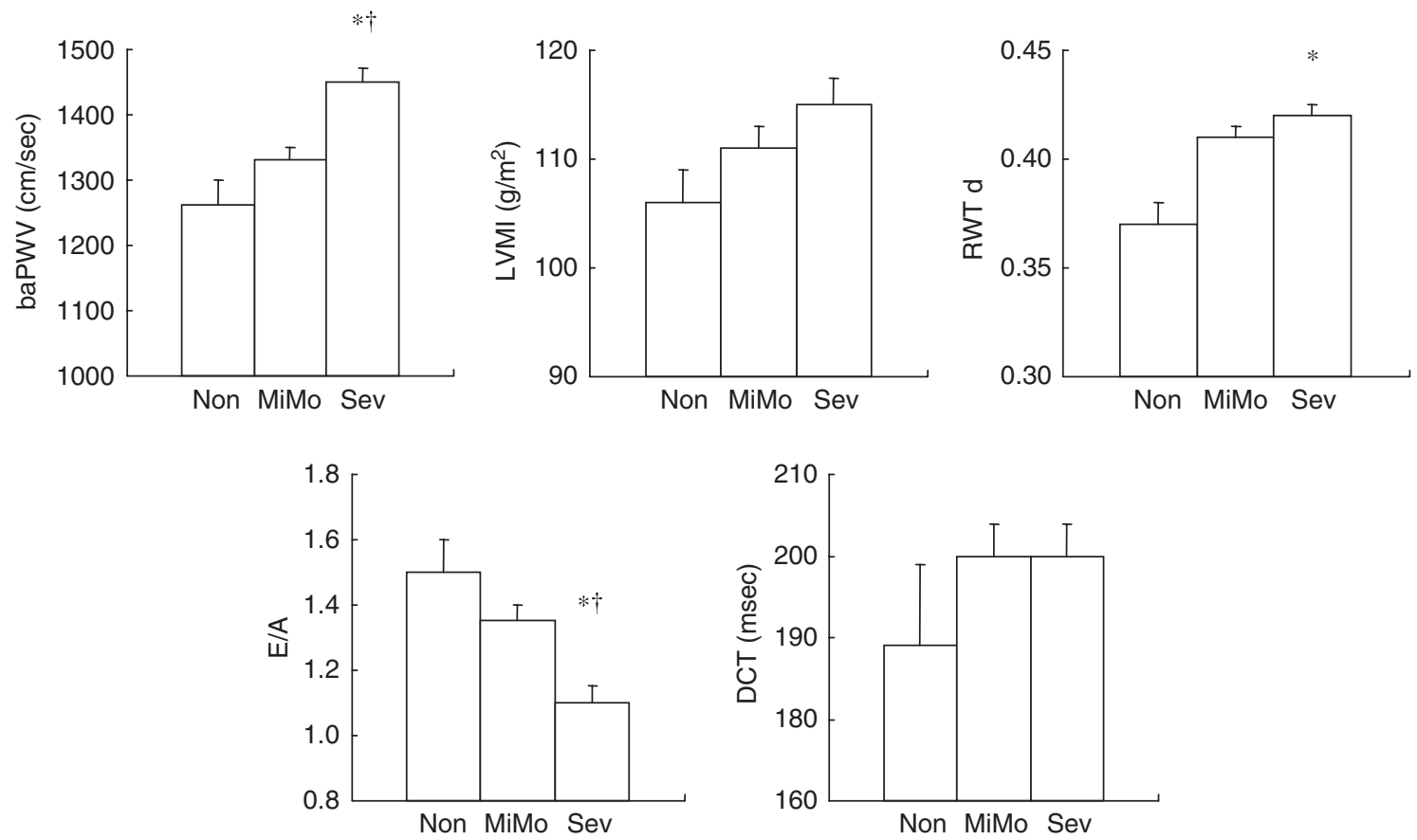

Figure 1 Brachial-ankle pulse wave velocity, left ventricular mass index, relative wall thickness, E/A ratio and deceleration time in the three groups classified by the severity of sleep apnea in subjects with normal clinic blood pressure. baPWV, brachial-ankle pulse wave velocity; LVMI, left ventricular mass index; RWTd, relative wall thickness at diastole; DCT, deceleration time; non, subjects without obstructive sleep apnea; MiMo, subjects with mild-to-moderate obstructive sleep apnea; Sev, subjects with severe obstructive sleep apnea; ${ }^{*} P<0.05$ vs subjects without obstructive sleep apnea without adjustments (for age, gender, smoking, body mass index, mean blood pressure, serum low-density lipoprotein cholesterol, serum high-density lipoprotein cholesterol, serum triglycerides, fasting plasma glucose and the presence of metabolic syndrome); ${ }^{\dagger} P<0.05$ vs subjects without obstructive sleep apnea with adjustments. 


\section{DISCUSSION}

To the best of our knowledge, no study has clearly indicated the existence of a relationship between arterial stiffening and the severity of OSA in subjects with normal clinic blood pressure. This study confirmed a significant relationship between the severity of OSA and arterial stiffness of the central to middle-sized arteries in such subjects. In OSA, in addition to the elevation of blood pressure and other obesity-related atherogenic factors (that is, obesity itself, dyslipidemia and abnormal glucose metabolism), metabolic syndrome, inflammation, increased oxidative stress and/or endothelial dysfunction are also noted, and these pathophysiological abnormalities are also thought to contribute to the structural arterial stiffening seen in these patients. ${ }^{1,2,10,11}$ Our earlier study indicated that OSA and metabolic syndrome were independently related to arterial stiffness of the central to middle-sized arteries. ${ }^{25}$ In the subjects who participated in this study, the severity of OSA was related to the arterial stiffness of the central to middle-sized arteries independent of the blood pressure level, other obesity-related atherogenic factors and the presence of metabolic syndrome. Therefore, although measurement of the nocturnal blood pressure level, which is known to be frequently elevated in subjects with OSA, was not conducted, the results of the study revealed a possible direct association between OSA and structural arterial stiffening in the central to middle-sized arteries, even in the absence of an obvious elevation of the clinic blood pressure.

Avelar et al. ${ }^{5}$ suggested the existence of an association between OSA and increased LV mass independent of the blood pressure level, and Arias et al. ${ }^{12}$ showed impairment of the cardiac diastolic function in normotensive OSA subjects. They proposed that nocturnal blood pressure elevation, increased sympathetic tone, intermittent hypoxia and large negative intrathoracic pressure changes during periods of airway obstruction might contribute to these OSA-related pressureindependent LV structural and functional changes. ${ }^{5,10,11,12}$ In the subjects of this study, although obesity causes LV structural and functional abnormalities, ${ }^{26}$ concentric LV remodeling and impairment of cardiac diastolic function were confirmed in severe OSA subjects. This impairment of cardiac diastolic function was significant even after adjustments for age, gender and risk factors for cardiovascular disease, including the body mass index. These abnormalities identify the patients at increased cardiovascular risk..$^{27,28}$ Furthermore, the baPWV was related to such LV abnormalities, independent of the severity of OSA and other adjusted variables (age, gender and risk factors for cardiovascular disease, including blood pressure). baPWV, which reflects the stiffness of the central to middle-sized arteries, has been shown to bear a close correlation with the aortic PWV and carotid-femoral PWV. ${ }^{23,29,30}$ The interaction between the increase in arterial stiffness and LV concentric remodeling, which may cause impairment of cardiac diastolic function, ${ }^{13,21,31}$ referred to as arterial-ventricular coupling, ${ }^{13,14}$ has been shown in normotensive subjects of advanced age. ${ }^{13}$ Considering the results of this study, we propose that the increased arterial stiffness of the central to middle-sized arteries associated with OSA may be related, at least in part, to the promotion of LV concentric remodeling, which may, in turn, be related to the impairment of cardiac diastolic function.

This study had some limitations. (1) A prospective study is proposed to confirm the contribution of OSA-related increase in the arterial stiffness to LV remodeling in subjects with normal blood pressure. (2) Central aorta is an important player of the ventriculararterial coupling. ${ }^{14}$ baPWV reflects the stiffness of the central to middle-sized arteries ${ }^{23,29,30}$ and, therefore, our results should confirm the use of carotid-femoral PWV, the gold standard for assessment of the central arterial stiffness. ${ }^{32}$ (3) In this study, the relationships among the severity of OSA, arterial stiffness of the central to middle-sized arteries, and LV structural and functional abnormalities were adjusted for blood pressure levels measured in the office setting. Although the elevation of nocturnal blood pressure has been reported with an increase in the severity of OSA, ${ }^{33}$ we could not assess the influence of nocturnal blood pressure on these relationships.

\section{CONCLUSION}

We showed the existence of increased arterial stiffness of the central to middle-sized arteries and LV structural/functional abnormalities in severe OSA patients with normal clinic blood pressure, suggesting the possible existence of a direct association between OSA and cardiovascular abnormalities. Possibly, arterial-ventricular coupling might be one of the underlying mechanisms of the concomitant existence of these cardiovascular abnormalities. The findings underscore the importance of careful evaluation of even severe OSA subjects with normal clinic blood pressure for cardiovascular abnormalities.

\section{ACKNOWLEDGEMENTS}

We are indebted to Professor J Patrick Barron of the International Medical Communications Center of Tokyo Medical University for reviewing this manuscript.

\section{CONFLICT OF INTEREST}

None declared.

1 Bassetti CL, Milanova M, Gugger M. Sleep-disordered breathing and acute ischemic stroke: diagnosis, risk factors, treatment, evolution, and long-term clinical outcome. Stroke 2006; 37: 967-972.

2 Cassar A, Morgenthaler TI, Lennon RJ, Rihal CS, Lerman A. Treatment of obstructive sleep apnea is associated with decreased cardiac death after percutaneous coronary intervention. J Am Coll Cardiol 2007; 50: 1310-1314.

3 Drager LF, Bortolotto LA, Figueiredo AC, Krieger EM, Lorenzi GF. Effects of continuous positive airway pressure on early signs of atherosclerosis in obstructive sleep apnea. Am J Respir Crit Care Med 2007; 176: 706-712.

4 Tsioufis C, Thomopoulos K, Dimitriadis K, Amfilochiou A, Tousoulis D, Alchanatis M, Stefanadis C, Kallikazaros I. The incremental effect of obstructive sleep apnoea syndrome on arterial stiffness in newly diagnosed essential hypertensive subjects. J Hypertens 2007; 25: 141-146.

5 Avelar E, Cloward TV, Walker JM, Farney RJ, Strong M, Pendleton RC, Segerson N, Adams TD, Gress RE, Hunt SC, Litwin SE. Left ventricular hypertrophy in severe obesity: interactions among blood pressure, nocturnal hypoxemia, and body mass. Hypertension 2007; 49: 34-39.

6 Cloward TV, Walker JM, Farney RJ, Anderson JL. Left ventricular hypertrophy is a common echocardiographic abnormality in severe obstructive sleep apnea and reverses with nasal continuous positive airway pressure. Chest 2003; 124: 594-601.

7 Safar ME, Levy BI, Struijker-Boudier H. Current perspectives on arterial stiffness and pulse pressure in hypertension and cardiovascular diseases. Circulation 2003; 107: 2864-2869.

8 Susic D, Frohlich ED. The aging hypertensive heart: a brief update. Nat Clin Pract Cardiovasc Med 2008; 5: 104-110.

9 Drager LF, Bortolotto LA, Figueiredo AC, Silva BC, Krieger EM, Lorenzi-Filho G. Obstructive sleep apnea, hypertension, and their interaction on arterial stiffness and heart remodeling. Chest 2007; 131: 1379-1386.

10 Parati G, Lombardi C, Narkiewicz K. Sleep apnea: epidemiology, pathophysiology, and relation to cardiovascular risk. Am J Physiol Regul Integr Comp Physiol 2007; 293: R1671-R1683.

11 Shamsuzzaman AS, Gersh BJ, Somers VK. Obstructive sleep apnea: implications for cardiac and vascular disease. JAMA 2003; 290: 1906-1914.

12 Arias MA, García-Río F, Alonso-Fernández A, Mediano O, Martínez I, Villamor J. Obstructive sleep apnea syndrome affects left ventricular diastolic function: effects of nasal continuous positive airway pressure in men. Circulation 2005; 112: 375-383.

13 de Simone G, McClelland R, Gottdiener JS, Celentano A, Kronmal RA, Gardin JM. Relation of hemodynamics and risk factors to ventricular-vascular interactions in the elderly: the Cardiovascular Health Study. J Hypertens 2001; 19: 1893-1903.

14 Kass DA. Age-related changes in venticular-arterial coupling: pathophysiologic implications. Heart Fail Rev 2002; 7: 51-62.

15 Rechtschaffen A, Kales A. A Manual of Standardized Terminology, Techniques and Scoring Systems for Sleep Stages of Human Subjects. UCLA Brain Information Service/ Brain Research Institute: Los Angeles, 1968.

16 American Sleep Disorders Association. EEG arousals: scoring rules and examples. Sleep 1992; 15: 173-184. 
17 Sleep-related breathing disorders in adults: recommendations for syndrome definition and measurement techniques in clinical research. The report of an American Academy of Sleep Medicine Task Force. Sleep 1999; 22: 667-689.

18 Johns MW. A new method for measuring daytime sleepiness: the Epworth sleepiness scale. Sleep 1991; 14: 540-545.

19 Teichholz LE, Kreulen T, Herman MV, Gorlin R. Problems in echocardiographic volume determinations: echocardiographic-angiographic correlations in the presence of absence of asynergy. Am J Cardiol 1976; 37: 7-11.

20 Devereux RB, Reichek N. Echocardiographic determination of left ventricular mass in man. Anatomic validation of the method. Circulation 1977; 55: 613-618.

21 Roman MJ, Ganau A, Saba PS, Pini R, Pickering TG, Devereux RB. Impact of arterial stiffening on left ventricular structure. Hypertension 2000; 36: 489-494.

22 Whalley GA, Walsh HJ, Gamble GD, Doughty RN. Comparison of different methods for detection of diastolic filling abnormalities. J Am Soc Echocardiogr 2005; 18: 710-717.

23 Yamashina A, Tomiyama H, Takeda K, Tsuda H, Arai T, Hirose K, Koji Y, Hori S, Yamamoto $Y$. Validity, reproducibility, and clinical significance of noninvasive brachialankle pulse wave velocity measurement. Hypertens Res 2002; 25: 359-364.

24 The Committee of Establishing the Definition of the Diagnosis of Metabolic Syndrome in Japan. J Jpn Soc Int Med 2005; 94: 188-203 (in Japanese).

25 Shiina K, Tomiyama H, Takata Y, Usui Y, Asano K, Hirayama Y, Nakamura T, Yamashina A. Concurrent presence of metabolic syndrome in obstructive sleep apnea syndrome exacerbates the cardiovascular risk: a sleep clinic cohort study. Hypertens Res 2006; 29: 433-441.
26 McGavock JM, Victor RG, Unger RH, Szczepaniak LS. Adiposity of the heart, revisited. Ann Intern Med 2006; 144: 517-524.

27 Muiesan ML, Salvetti M, Monteduro C, Bonzi B, Paini A, Viola S, Poisa P, Rizzoni D, Castellano M, Agabiti-Rosei E. Left ventricular concentric geometry during treatment adversely affects cardiovascular prognosis in hypertensive patients. Hypertension 2004; 43: 731-738.

28 Schillaci G, Pasqualini L, Verdecchia P, Vaudo G, Marchesi S, Porcellati C, de Simone $G$, Mannarino E. Prognostic significance of left ventricular diastolic dysfunction in essential hypertension. J Am Coll Cardiol 2002; 39: 2005-2011.

29 Munakata M, Ito N, Nunokawa T, Yoshinaga K. Utility of automated brachial ankle pulse wave velocity measurements in hypertensive patients. Am J Hypertens 2003; 16: 653-657.

30 Sugawara J, Hayashi K, Yokoi T, Cortez-Cooper MY, DeVan AE, Anton MA, Tanaka H. Brachial-ankle pulse wave velocity: an index of central arterial stiffness? J Hum Hypertens 2005; 19: 401-406.

31 Shapiro BP, Lam CS, Patel JB, Mohammed SF, Kruger M, Meyer DM, Linke WA, Redfield MM. Acute and chronic ventricular-arterial coupling in systole and diastole: insights from an elderly hypertensive model. Hypertension 2007; 50: 503-511.

32 Guidelines for the Management of Arterial Hypertension. The Task Force for the Management of Arterial Hypertension of the European Society of Hypertension (ESH) and of the European Society of Cardiology (ESC). J Hypertens 2007; 25: 1105-1187.

33 Bazzano LA, Khan Z, Reynolds K, He J. Effect of nocturnal nasal continuous positive airway pressure on blood pressure in obstructive sleep apnea. Hypertension 2007; 50: 417-423. 